\title{
Micro, Meso, and Macro Data Collection and Analysis, as a Method for Speculative and Artistic Exploration
}

\author{
Stavros Didakis \\ School of Art, Design, and Architecture, University of Plymouth \\ Plymouth, PL48AA, United Kingdom \\ stavros.didakis@plymouth.ac.uk
}

\begin{abstract}
In this work, an attempt is made to explore the emerging computationally-enhanced private and public environments by analysing the ecological transitions and their implications on practical, aesthetic, and speculative dimensions. The author has decided to methodologically dissect the multiplicity of information that exists on many possible-to-detect scales, that is, micro (10-10 to 103), meso (10-3 to 101), and macro (101 to 108), and utilize this extraction as a tool for experimentation and redefinition. With the use of custom-made hardware and software utilities (sensor devices, sentiment analysis algorithms, online APIs, and many more), a vast amount of data is collected and used as a multidimensional layered architecture that constantly shifts and transforms. The extracted and analysed content of the collection becomes the essence of the work that is shaped and refined through digital and physical making - middleware, recursion, mapping and by utilizing technological objects within the physical space, the creative process is augmented and amplified, exploring not only new practices and novel applications, but rather redefining behaviour, thought-process, and context.
\end{abstract}

Ecology. Cybernetics. Data. Sensing. Internet of Things. Mapping

\section{INTRODUCTION}

Our current almighty techno-gods have successfully managed to deliver their utopian promises; immediacy, satisfaction, engagement. Planetary consciousness is now a screen away, allowing us to instantly access millions of petabytes on a blink of an eye. The manifested reality is now enhanced, augmented, and glitched, offering to the creative mind saturated desires that redefine the limitations of our bodies, consciousness, and imagination. The repercussions have dwelled the core of human existence, reshaping as well our understanding of what Art is, or what it can ultimately become.

Examining this transitional phase in our post-digital societies, trends and patterns begin to flourish and reproduce, demonstrating that the artistic exploration is not limited to traditional tools and resources, but spans across electronics, digital media, artificial and evolutionary systems, virtual cyberspaces, as well as organisms, bodies, and cells. The new ecologies that are formed radically expand on multiple dimensions, generating uncharted cartographies that invite for exploration and experimentation.
According to this reason, laws of ecosystems are being used to contextualize the techno-ecologies that are formed by the functions and behaviours of the computational machines. The new agents within our environments cause the emergence of new systems, concepts, layers, and maps that relate to our understanding of possibilities in conceptual, functional, and creative dimensions. Due to this reason, a multi-layered architecture is suggested here, to assist in the definition of a framework both for the categorization of information and systems, but also to the practical exploration of these layers within an explorative and speculative development approach, with an objective to create a methodological analysis in approaching our constantly-shifting hybrid spaces.

\section{RESILIENCE AND STABILITY}

In 1973, Crawford S. Holling (1973) published a paper on the resilience and stability of ecological systems, where he presented his findings on the behaviour of ecologies that is affected by external events of multiple levels of criticality. Holling was interested in observing how external forces impact 


\section{Stavros Didakis}

the state of dynamic equilibrium, that is, how these forces cause fluctuations both in amplitude and frequency in relation to the parameters of the ecological system. According to the level of resonances, the state of equilibrium can be destabilized, and in some cases fractured or even destroyed.

In relation to the discussion of natural systems, this can be clearly illustrated by the impact of human activity on the environment and its biosphere, which sets off balance the natural order. With the extreme overtake of natural resources and the production of destructive and deadly chemicals that pollute soil, ground, and waters, the stability of multiple eco-systems is threatened and greatly endangered. However, even though humans dramatically change the states of equilibrium in many cases, Holling suggests these wide amplitudes of fluctuations cause repercussive effects that establish new domains of attraction. Even when the ecology reaches and over-exceeds a critical limit, adaptive shifts emerge, which is a natural way for the system to survive and evolve.

Holling noticed that even when the biosphere is largely instable due to large fluctuations that are caused by an external force, it "may introduce a resilience and a capacity to persist" (Holling 1973: 15), as the system exercises its reflexes and responds accordingly. It is worth mentioning, that, on the other hand, the survival of the system is not guaranteed if no forces attack the ecosystem; "a stable maximum sustained yield of a renewable resource might so change these deterministic conditions that the resilience is lost or reduced so that a chance and rare event that previously could be absorbed can trigger a sudden dramatic change and loss of structural integrity of the system" ( $p$. 21).

\section{TECHNO-ECOLOGIES AND ADAPTIVE SHIFTS}

The phenomena observed on the dynamic resonances that are caused by the computational technologies on the substrata of the human experience, directly relates to Holling's studies on the resilience and stability of ecological systems. The understanding of our Umwelt (and what constitutes our constructed reality), is greatly influenced by the cybernetic surrounding, as within the current hybrid spaces that we occupy physical, virtual, augmented - adaptive shifts take place, and radical changes in the routines, rhythms, and properties of our lifestyle manifest due to the technological development that redefines the human understanding and nature.
Humberto Maturana and Francisco Varela in their studies related to autopoiesis and cognition (1980) claim that a "structural coupling" is formed between "a living system's connection to its environment, and the mutual communicative connections between the two systems" (p. 24). If we accept this statement as true, then we can also accept that there is a structural coupling with the cognitive and perceptual connections between ourselves and our computationally-enhanced environment, which directly means that our Umwelt has shifted due to the digital realities that have penetrated our private ecosystems with a great force. This is also reflected by the premise of Martin Dodge and Rob Kitchin that computational processes are not only built into the objects of our surrounding environments, but into the fabric of dwelling itself (2008).

The techno-environment is in a state of flux, as the multiple agents that exist on its "pragmasphere or functional universe" (Sharov 2010) such as inorganic, biological, digital, all strive to achieve a dynamic equilibrium. In spite of the multiple destabilizing forces that are exerted, the ecosystem seems to become resilient enough to absorb these impacts and adjust itself to the new changes, and, in addition, result in new emergencies (creative, functional, aesthetic) when the equilibrium overexceeds its original limits.

The electrical control in the first half of the twentieth century, infected the mechanical public and personal space (Cowan 1983) in a similar way that electrical and electronic objects become "colonialized by computer code" (Dodge \& Kitchin 2008: 13). Ubiquitous computing (ubicomp), Ambient Intelligence (Aml), and the Internet of Things (loT) are practices within Computing Sciences that intend to spatialise digital interfaces and applications into the human-sphere in order to resolve complicated daily tasks, build practices that are context-aware and responsive to real-time needs, and also assist in monitoring and registering a range of information and interactions.

Algorithms, machine intelligence, and deep learning are already part of our daily lexicon and their solid establishment is directly reflected from the speed and wide array of developments that take place. The new computational landscape, has not only affected our public and personal ecosystems, but have also created multidimensional possibilities in sensing, collecting, and identifying information from visible and invisible spectra. The patterns extracted from the unseen choreographies of our daily lives, provide rich information for the accumulation of assembled events that assist in constructing knowledge and further understanding. 


\section{MULTI-LAYERED ARCHITECTURES}

"The map is open and connectable in all of its dimensions; it is detachable, reversible, susceptible to constant modification. It can be torn, reversed, adapted to any kind of mounting, reworked by an individual, group, or social formation. It can be drawn on a wall, conceived of as a work of art, constructed as a political action or as a meditation." (Deleuze \& Guattari 1987: 12)

Gilles Deleuze and Felix Guattari in their book "A Thousand Plateaus" explain the concept of rhizome, an image of a thought process that consists of assemblages of dissimilar properties such as "words, things, power, and geography" (Deleuze \& Guattari 1987: 25). The assemblages are able to organize themselves in non-hierarchical lateral networks, and to develop their own experimental intelligence by creating new and heterogeneous connections between them. The rhizome is never static but always changes form, characteristics, and properties, according to new assembled connections - becoming infinitely multidimensional.

Benjamin $\mathrm{H}$. Bratton, inspired by the studies of Deleuze \& Guattari, attempts to illustrate the geopolitical architectures of complex computational structures, considering the combination of their properties as a "metaplatform of an alternative counterindustrialization" (Bratton 2016: 96). His proposed model, the Stack, consists of six elements (User, Interface, Address, City, Cloud, Earth) where each one of them contain their own computational dimensions, however, they are all interconnected - having the network act as an "armature of the social itself" (p. 19) - and allow us to conceive an informational megastructure as an ultimate cybernetic system.

The approach taken in this research relates to the aforementioned concepts and frameworks, although the system that has been implemented here intends to extend our understanding both conceptually and practically in regards to the mapping of properties that exist on micro, meso, and macro scales of the public and private environments we occupy. Within these spaces, a vast number of unseen cosmoses exist that calibrate and redefine (many times unconsciously or imperceptibly) experience, functions, aesthetics, context, as well as all possible relationships between them. The ability to perceive the environment in all dimensions and the hidden properties and relationships between objects, spaces, digits, bodies, and multi-layered ecologies, reveals an underlying multitude of processes and a swarm of functions that define the very essence of our Umwelt and how reality is abstracted, reasoned, and perceived.

Thus, for the needs of this work, an array of sensing agents has been implemented on physical and digital realms with main intention to identify properties and events on micro, meso, and macro scales. Micro refers to the microscopic scale of the surrounding environment that contains properties that cannot be easily perceived by the human senses and these include matter and substances such as bacteria, chemicals, particulate and biological molecules, radioactive and organic pollutants, radon, carbon monoxide, lead, mercury, and carcinoid elements. Microscopic substances are able to penetrate live tissue and be absorbed by human organs, affecting the body in multiple ways - transforming and shifting on a cellular level its development, growth, and evolution. It has been already established that the substances can cause a range of symptoms such as asthma, respiratory dysfunctions, nausea, suffocation, infections, damage to lung tissue, cardiovascular disease, chronic lung disease, neurological impairments, cancer, premature death, and many more (EPA 2007). For this reason, a sensing prototype has been developed by the author to thoroughly analyse and log 20 different airborne properties in order to provide a tangible understanding of an array of chemicals, molecules, and particulates that exist within the monitored space.

In addition to the properties on the micro scale, a range of elements on both the physical and cyber space are examined, which are categorized in the meso scale, that is, the intermediate scale between micro and macro, and includes events and interactions of peoples' physical and virtual preferences, activities and presence (Moeslund \& Granum 2001), behaviors, as well as biological and emotional responses (Nasukawa \& Yi 2003, Biondi et al. 2017). With the development and implementation of sensing systems (both on the physical and digital space), bio-tracking interfaces, and software utilities (logging systems, sentiment analysis algorithms, social media content), a large quantity of information is collected and used to comprehend in more detail aspects, patterns, rhythms, and interactions that take place within the examined ecology.

Every ecosystem is constantly affected by macro events, such as planetary, climate, geomorphological, or weather conditions. For example, momentary affective states (e.g. those produced by the weather) influence judgments about happiness and satisfaction (Schwarz \& Clore 1983, Catalano \& Dooley 1977), the moon's gravity causes tides that affect human behaviour (Govorushko 2012), and Ultraviolet Index (UV) produces or initiates carcinogenesis in the human 


\section{Stavros Didakis}

skin (Wilkening 2006). Concerning the importance of macro events, the Millennium Ecosystem Assessment Board notes:

\begin{abstract}
"There are natural and human-induced physical, chemical, and biological drivers of change. Natural drivers include solar radiation, climate variability and extreme weather events (such as droughts, floods, hurricanes, and cyclones), fires, volcanic eruptions, earthquakes, pest and disease out- breaks, and natural biological evolution. The primary human-induced drivers include land use changes, climate change, air and water pollution, acid deposition, soil erosion, soil salinization and fertility changes, irrigation, fertilizer use, harvesting, the use of persistent organic chemicals, and the introduction of non-native species". (Millennium Ecosystem Assessment Board 2003)
\end{abstract}

Furthermore, social, political, economic events, fluctuations in the stock market, crude oil price indices and major events in media and popular culture reflect significant, immediate and highly specific effects on various dimensions of people's mood and their habitats (Bollen et al. 2010, Lefebvre 1991, Schöning \& Bonhage 2015). As all of the above environmental properties are extremely important for the ecology, a set of computational processes have been configured to collect information from open-access real-time communication systems, such as Really Simple Syndication (RSS) feeds and Application Programming Interfaces (APIs), which provide information on weather conditions, Ultraviolet and Air Quality Index (AQI), and data on earthquakes and solar radiation.

The information collected in this research project from the aforementioned micro, meso, and macro scales is stored, analysed, and visualized, with a main goal to identify overlaps, cross-overs, or contrasted data sets that can be highly relevant in understanding this emerging rhizome. Although the properties collected provide only a portion of the whole "stack", they nevertheless become an indispensable part of the collected and ecological consciousness that enhance our awareness of the unseen relationships that have a substantial impact on physical and biological entities, altering and defining material and immaterial structures.

\section{CREATIVE ABSTRACTIONS}

As William Mitchell argues, "networked intelligence is being embedded everywhere, in every kind of physical system-both natural and artificial", and "routinely, events in cyberspace are being reflected in physical space, and vice versa" (Mitchell 2003: $3)$. The collected information from all sensor agents described above becomes an integral part of the ecology, and the cloud is now a fundamental medium for the manipulation of captured instances, allowing a range of processing possibilities to emerge through modular applications, and offering a creative palette for the design of computationallyenhanced meta-processes.

By developing middleware, machine learning algorithms, and personalized user interfaces, we do not only have a way to analyse and visualise the captured content (A/B testing, association rule learning, classification and clustering, sentiment analysis, data mining), but more importantly, we are able to extend the creative potential of the system and explore the possibilities in binding data and properties of physical, virtual, and augmented spaces, as well as context, media layers, and invisible matter. This process provides a compositional framework for orchestrating responses, interactions, and dynamic interrelationships between properties and agents.

A series of speculative artefacts have been developed for this work to realize these possibilities and experiment with the mapping of data inputs, processing algorithms, and output media. This has resulted to a number of practices such as lighting compositions, augmented visualizations, responsive surfaces, automated mechanics, tangible interactions, reconfigurable structures, hybrid objects, sonified environments. A fundamental criterion for these developments was to use the information extracted from the micro, meso, and macro spaces as a way for defining generative content and utilize this as a creative medium that becomes inseparable from the nature of the ecosystem itself. The flexibility offered by computational media technologies to assist in the creation of shiftable and expressive spaces, offers the possibilities for the ecology to reflect on its current (or hetero-chronological) aspects, not only regarding individual quantitative and qualitative data, but also to comprehend relationships between properties and how larger clusters are formed.

The creative exploration of the networked and media apparatuses is an integral part of the ecology's regulation mechanism to achieve homeostasis, a term coined by Norbert Wiener in 1920s to describe "the body's organic mechanism for maintaining healthy, stable states of internal equilibrium through a balance of self-regulating actions and reactions" (Conway and Siegelman 2005: 16). The multidimensional dynamics of all consisting agents has to be abstracted in an imaginative way to be cognitively understood, in a 
similar way Socrates would dissect on knowledge and facts, aiming at seizing the unity in the multiplicity of the real (Plato 1986).

\section{CONCLUSION}

This paper describes main aspects of a theoretical and practical framework that has been developed by the author to methodologically approach the cartography, analysis, and exploration of our current hybrid environments by utilizing a set of physical, augmented, and digital systems and processes to reorganize and define the mapping architecture in micro, meso, and macro scales. Natural ecologies and their mathematical representations have been studied to assist in understanding the redefinition of our techno-spaces considering the computational processes as functional agents that radically affect our surroundings and ourselves. This transitional phase is accompanied with threads and opportunities, forcing us to consider the development of conceptual maps and frameworks so that we comprehend this uncharted multi-dimensionality, and develop a critical and perceptual stance on the hybridization of validated, augmented, and virtual realities.

\section{REFERENCES}

Biondi, G., Franzoni, V., and Poggioni, V. (2017) A Deep Learning Semantic Approach to Emotion Recognition Using the IBM Watson Bluemix Alchemy Language. In Gervasi, O. et al. (eds.) Computational Science and Its Applications ICCSA 2017. Lecture Notes in Computer Science, vol. 10406. Springer, Cham, 718-729.

Bollen, J., Pepe, A., and Mao, H. (2010) Modelling Public Mood and Emotion: Twitter Sentiment and Socio-Economic Phenomena. In Proceedings of the Fifth International AAAI Conference on Weblogs and Social Media, Association for the Advancement of Artificial Intelligence, 450-53.

Bratton, B. H. (2016) The Stack: On Software and Sovereignty. MIT Press: Cambridge, MA.

Catalano, R., and Dooley, C. D. (1977) Economic Predictors of Depressed Mood and Stressful Life Events in a Metropolitan Community. In Journal of Health and Social Behavior, 18, 292-307.

Conway, F., and Siegelman, J. (2005) Dark Hero of the Information Age. In Search of Norbert Wiener the Father of Cybernetics, Basic Books: Cambridge, MA.

Cowan, R. S. (1983) More Work for Mother: The Ironies of Household Technology from the Open Hearth to the Microwave. Basic Books: New York.
Deleuze, G., and Guattari, F. (1987) A Thousand Plateaus. Translated by B. Massumi, University of Minnesota Press: Minneapolis.

Dodge, M., and Kitchin, R. (2008) Software, Objects and Home Space. Environment and Planning A, August, 1-36.

EPA Environmental Protection Agency (2007) Health Effects of Air Pollution. http://www.epa.gov/region07/air/quality/health.htm (retrieved 5 October 2017).

Govorushko, S. M. (2012) Natural Processes and Human Impacts. Interactions between Humanity and the Environment. Springer: Dordrecht, Heidelberg, London, New York.

Holling, C. S. (1973) Resilience and Stability of Ecological Systems. In Annual Review of Ecology and Systematics, 4, 1-23.

Lefebvre, H. (1991) The Production of Space. Blackwell: Cambridge.

Maturana, H. R., and Varela, F. (1980) Autopoiesis and Cognition: The Realization of the Living. Reidel: London.

Mitchell, W. J. (2002) ME++ The Cyborg Self and the Networked City. MIT Press: Cambridge, MA.

Moeslund, T. B., and Granum, E. (2001) A Survey of Computer Vision-Based Human Motion Capture. In Computer Vision and Image Understanding, 81(3), 231-68.

Nasukawa, T., and Yi, J. (2003) Sentiment Analysis: Capturing Favorability Using Natural Language Processing. In Proceedings of the 2nd International Conference on Knowledge Capture, New York: ACM, 70-7.

Plato (1986) The Dialogues of Plato. Bantam Dell: New York.

Schöning, J., and Bonhage, C. E. (2015) On the Acquisition of Human Emotions in Space and Time. In Biljecki, F. \& Tourre V. (eds.) Eurographics Workshop on Urban Data Modelling and Visualisation, 49-54.

Schwarz, N., \& Clore, G. L. (1983) Mood, Misattribution, and Judgments of Well-Being: Informative and Directive Functions of Affective States. In Journal of Personality and Social Psychology, 45, 513-23.

Sharov, A. A. (2010). Functional Information: Towards Synthesis of Biosemiotics and Cybernetics. In Entropy, Basel, Switzerland, 12(5), 1050-1070.

Wilkening, G. M. (2006) Non-lonizing Radiations. In Pfafflin, J. R. \& Ziegler, E. N. (eds.) Encyclopedia of Environmental Science and Engineering, 1(A-L), 5th Edition, Taylor \& Francis: Raton, London, New York, 779-90. 